\title{
Dark Matter indirect detection: recent developments and perspectives
}

\author{
Marco CIRELLI ${ }^{* \dagger}$ \\ IPhT CNRS/CEA Saclay \\ E-mail: marco.cirelli@cea.fr
}

I discuss two recent anomalies in Dark Matter Indirect Detection (the positron excess and the 130

$\mathrm{GeV}$ line), some relevant constraints and theory developments.

SACLAY-T14-186

Proceedings of the Corfu Summer Institute 2013,

31 August - 11 September 2013

Corfu, Greece

\footnotetext{
* Speaker.

$\dagger$ I thank my collaborators on the papers which led to the results presented here and who helped me with many useful discussions. I acknowledge the hospitality of the Institut d'Astrophysique de Paris, where part of this work was done. Funding and research infrastructure acknowledgements: European Research Council (ERC) under the EU Seventh Framework Programme (FP7/2007-2013)/ERC Starting Grant (agreement n. 278234 — 'NEWDARK' project); French national research agency ANR under contract ANR 2010 BLANC 041301.
} 


\section{Introduction}

Indirect searches for Dark Matter (DM) aim at detecting the signatures of the annihilations or decays of DM particles in the fluxes of Cosmic Rays (CRs), intended in a broad sense: charged particles (electrons and positrons, protons and antiprotons, deuterium and antideuterium), photons (gamma rays, X-rays, synchrotron radiation), neutrinos. In general, a key point of all these searches is to look for channels and ranges of energy where it is possible to beat the background from ordinary astrophysical processes. This is for instance the basic reason why searches for charged particles focus on fluxes of antiparticles (positrons, antiprotons, antideuterons), much less abundant in the Universe than the corresponding particles, and searches for photons or neutrinos have to look at areas where the DM-signal to astro-noise ratio can be maximized (typically the Galactic Center and DM-dominated structures such as dwarf satellite galaxies).

Pioneering works have explored indirect detection (ID) as a promising avenue of discovery since the late-70's. Since then, innumerable papers have explored the predicted signatures of countless particle physics DM models. In the past 6 years or so, however, the field has experienced a significant burst of activity, mainly due to the results presented by a few very well performing experiments, above all the PAMEla satellite, the FERmi satellite and the Hess telescope. It is fair to say that the field has passed, for better or for worse, from a theory-driven state to a data-driven phase.

In this presentation I intend to briefly review the current status of the field, using the pretext of discussing two recent experimental 'anomalies' and the ensuing phenomenological activity. The two anomalies are: 1) the positron and electron excesses, first soundly detected by PAMELA in 2008 in the positron fraction and then corroborated by many results from FERMI, HESS and recently AMS-02; 2) the ' $130 \mathrm{GeV}$ line' from the Galactic Center (GC), first identified in 2012 by Christoph Weniger and collaborators in FERMI data.

\section{The positron and electron excesses}

There has been a flurry of positive results from a few indirect detection experiments looking at the fluxes of charged cosmic rays. In particular, the signals pointed to an excess of electrons and positrons at the $\mathrm{TeV}$ and sub- $\mathrm{TeV}$ scale:

- Notorius data from the PAMELA satellite [1] showed, back in 2008, a steep increase in the energy spectrum of the positron fraction $e^{+} /\left(e^{+}+e^{-}\right)$above $10 \mathrm{GeV}$ up to $100 \mathrm{GeV}$, compatibly with previous hints from HEAT [2] and AMS-01 [3]. These findings have later been confirmed with independent measurements by the FERMI satellite [4] and, recently, by the AMs-02 experiment [5] and extended to about $430 \mathrm{GeV}$.

- Data from Pamela [6] also showed no excess in the $\bar{p}$ energy spectrum compared with the predicted background.

- In the $e^{+}+e^{-}$energy spectrum, the results of the FERMI satellite [7], combined with the results from the HESS telescope [8], hint to an excess (with respect to the expected background) reproduced by a simple power law up to about $1 \mathrm{TeV}$ and eventually a steepening at energies of a few TeVs. 

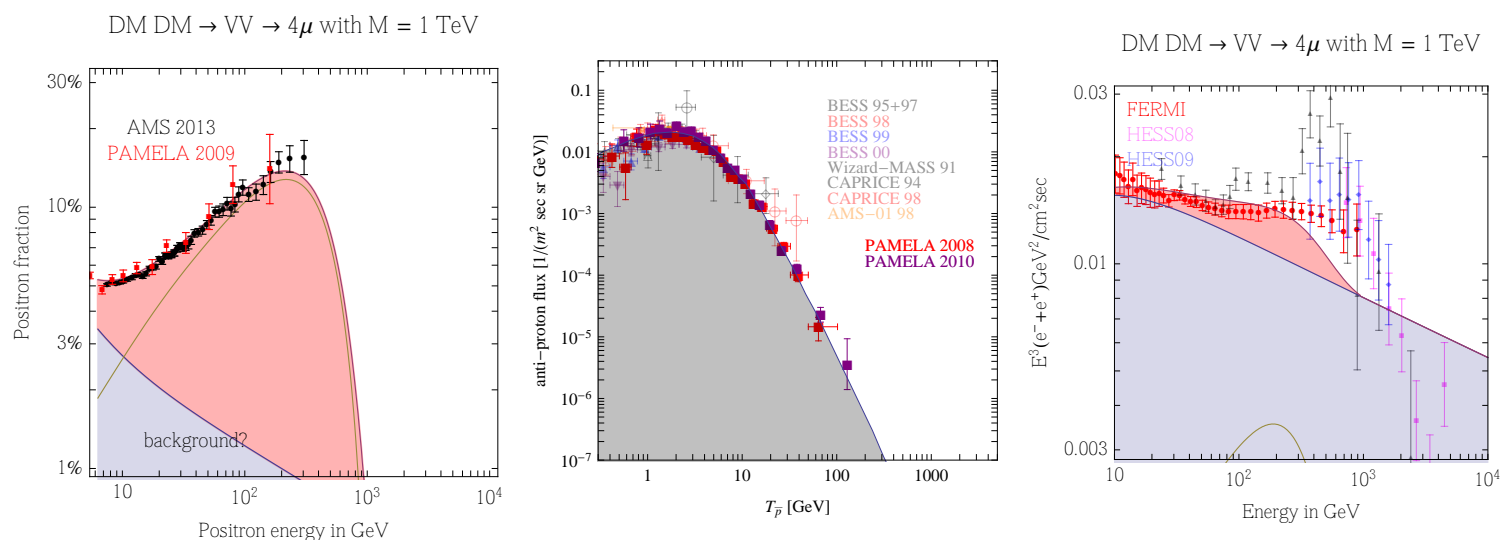

Figure 1: A compilation of recent and less recent data in charged cosmic rays, superimposed on plausible but uncertain astrophysical backgrounds from secondary production and on the flux produced by Dark Matter annihilations for a specific model. Left: positron fraction. Center: antiproton flux. Right: sum of electrons and positrons. Figures from ref. [9].
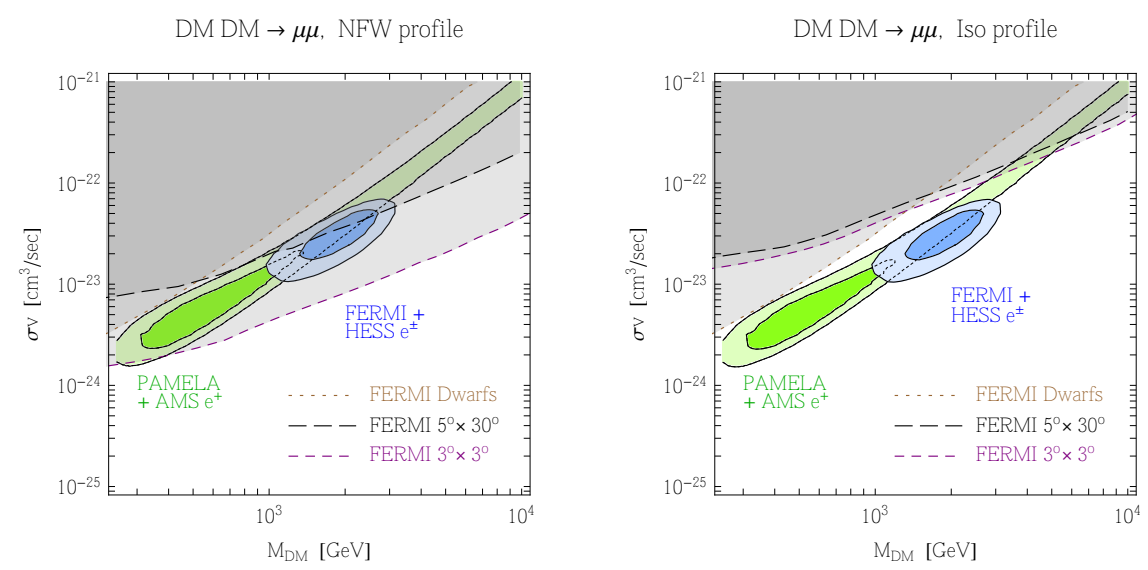

Figure 2: Best fit regions for the positron and electron excesses, together with some representative $\gamma$-ray constraints. Figure from ref. [9].

The data are displayed in fig. 1, together with the expected astrophysical 'backgrounds' and with the contribution from an annihilating DM particle which fits them reasonably well (see below). The properties of such a particle are pin-pointed quit precisely by the data. The DM has to be:

$\triangleright$ With a mass of 1 to few $\mathrm{TeV}$, in order to reproduce the feature in the $e^{+}+e^{-}$spectrum. Actually, the hint of a flattening in the positron fraction suggested by AMS-02 favours a DM mass below about $1 \mathrm{TeV}$ with about $3 \sigma$ statistical significance, depending on the DM annihilation channel, so that a little bit of a tension is present with the $e^{+}+e^{-}$spectrum, which requires a slightly larger value.

$\triangleright$ Leptophilic, i.e. annihilating almost exclusively into leptonic channels, otherwise the antiproton measurements would be exceeded.

$\triangleright$ With a very large annihilation cross section, of the order of $10^{-23} \mathrm{~cm}^{3} / \mathrm{sec}$ or more (for the masses under consideration), much larger than the thermal one, in order to produce a large 

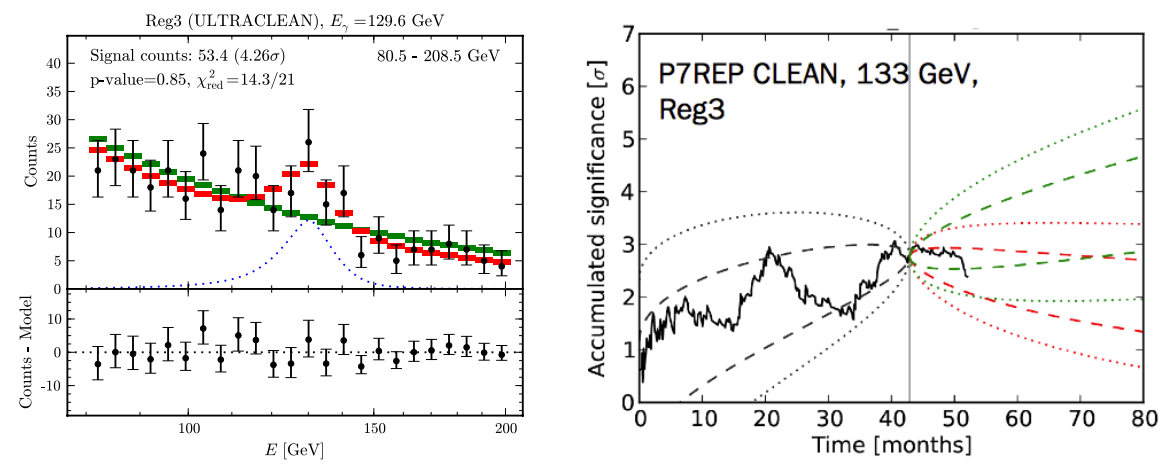

Figure 3: Left: FERMI $\gamma$-ray data and fits pointing to a line at about $130 \mathrm{GeV}$. Right: behavior with time of the accumulated significance for this signal. Figures from ref. [15] and ref. [24].

enough flux that can fit the positron rise and the $e^{+}+e^{-}$bump.

As tantalizing as these hints of DM can be, they have to be confronted with associated constraints. Many possible constraints can be considered, but here I will focus on two classes only. The first one is observations of $\gamma$-rays. In fig. 2 we show representative $\gamma$-ray bounds (the constraints are taken from $[10,11]$, more recent analyses find similar or slightly more stringent bounds). We see that the fit region shows some tension with $\gamma$-ray data (or it is rather clearly excluded) if (left) we have chosen a benchmark NFW galactic Dark Matter profile. Choosing the shallower isothermal profile (right), however, makes the constraints looser. It is therefore difficult to get a final answer from $\gamma$-rays. The second class of constraints comes from observations of the cosmic microwave background (CMB), which imposes bounds on DM annihilations (based on the fact that they would have re-ionized the primordial universe) that disfavor at various degrees and for most channels the DM interpretation of the positron excess [12].

\section{The $130 \mathrm{GeV}$ line}

The ' $130 \mathrm{GeV}$ line' claim has gathered a lot of attention in the past two years (for a more thorough review see [13]). Originally spotted by [14] and, above all, by [15] in the publicly available FERMI data from an extended region including the GC (fig. 3 left reports the most evocative of the original analysis' figures), it has later found support in other analyses [16, 17, 18, 19], with varying degrees of accuracy and claimed significance. $[16,19]$ have seen it in what could possibly be DM subhaloes of the MW, and there might be two lines, at $111 \mathrm{GeV}$ and $129 \mathrm{GeV}$ [20, 17]. [18] has seen it in galaxy clusters too. For a response, [21, 22, 23] challenged the analyses in a number of ways, suggesting that the line(s) could be due to unidentified instrumental, statistical or astrophysical origin. Although it is probably too early for a final conclusion on this claim, it is fair to say that the current consensus seems to be that the line has been a rather unfortunate combination of an instrumental effect and a statistical fluctuation. The right panel of fig. 3 illustrates that, as more data are accumulated, the significance of the signal lowers, hence pointing at something which is probably not an actual signal. 


\section{Conclusions}

There are arguably no firm conclusions in this field at this moment in time. There are tantalizing hints (the positron and electron excess, the gamma-ray line and the the GeV GC excess from the Galactic Center) and there are stringent constraints. Such constraints, however, are often relaxed by appropriate assumptions, which can be extreme or not. The only firm albeit generic conclusions seem to be that:

$\diamond$ current experiments are clearly reaching (and in some cases have already reached) the sensitivities for which they were designed, and hence they probe very promising regions of the parameter space;

$\diamond$ astrophysics, in different manifestations, is the main killjoy, introducing alternative compelling explanation, irreducible uncertainties, unbeatable background noise...;

$\diamond$ hence, it is important to pursue a multi-messenger approach in all instances, investigating associated signals in other channels, cross-checking constraints and confirmations from independent targets etc;

$\diamond$ in any case, the profusion of data from the recent experiments have spurred a remarkable proliferation of DM models, so that 'traditional' DM models (such as SuSy DM) have, for better or for worse, been joined by many other possibilities.

\section{References}

[1] O. Adriani et al. [PAMELA Collaboration], Nature 458, 607-609, 2009, arXiv:0810.4995.

[2] S. W. Barwick et al. [HEAT Coll.], Astrophys. J. 482 (1997) L191 [astro-ph/9703192].

[3] M. Aguilar et al. [AMS-01 Coll.], Phys. Lett. B 646 (2007) 145 [astro-ph/0703154].

[4] M. Ackermann et al. [The Fermi LAT Collaboration], arXiv:1109.0521 [astro-ph.HE].

[5] M. Aguilar et al. [AMS Collaboration], Phys. Rev. Lett. 110 (2013) 141102.

(An updated measurement has only been presented in a talk by S. Ting (and subsequent talks elsewhere).)

[6] O. Adriani et al. [PAMELA Coll.], Phys. Rev. Lett. 105 (2010) 121101 [arXiv:1007.0821].

[7] A. Abdo et al. [Fermi-LAT Coll.], Phys. Rev. Lett. 102 (2009) 181101, arXiv:0905.0025.

M. Ackermann et al. [Fermi LAT Coll.], Phys. Rev. D 82 (2010) 092004, arXiv:1008.3999.

[8] F. Aharonian et al. [H.E.S.S. Coll.], Phys. Rev. Lett. 101 (2008) 261104 [arXiv:0811.3894].

F. Aharonian et al. [H.E.S.S. Coll.], Astron. Astrophys. 508 (2009) 561 [arXiv:0905.0105].

[9] M. Cirelli, M. Kadastik, M. Raidal and A. Strumia, Nucl. Phys. B 813 (2009) 1 [Addendum-ibid. B 873 (2013) 530] [arXiv:0809.2409 [hep-ph]].

[10] M. Cirelli, P. Panci and P. D. Serpico, Nucl. Phys. B 840 (2010) 284 [arXiv:0912.0663]. M. Papucci and A. Strumia, JCAP 1003 (2010) 014 [arXiv:0912.0742]. 
[11] Fermi-LAT collaboration, Phys. Rev. Lett. 107 (2011) 241302 [arXiv:1108.3546]. Fermi-LAT collaboration, Astrophys. J. 761 (2012) 91 [arXiv:1205.6474]. For this figure we used the dwarfs data presented by A. Drlica-Wagner at the 2012 Fermi symposium.

[12] S. Galli, F. Iocco, G. Bertone and A. Melchiorri, Phys. Rev. D 80 (2009) 023505 [arXiv:0905.0003]. T.R. Slatyer, N. Padmanabhan, D.P. Finkbeiner, Phys. Rev. D 80 (2009) 043526, 0906.1197. G. Huetsi, A. Hektor, M. Raidal, Astron. Astrophys. 505 (2009) 999, arXiv:0906.4550. M. Cirelli, F. Iocco and P. Panci, JCAP 0910 (2009) 009, arXiv:0907.0719. See also: T. Kanzaki, M. Kawasaki and K. Nakayama, Prog. Theor. Phys. 123 (2010) 853, arXiv:0907.3985. Q.Yuan, B.Yue, X.-J.Bi, X.Chen, X.Zhang, JCAP 1010 (2010) 023, arXiv:0912.2504. G. Hutsi, J. Chluba, A. Hektor and M. Raidal, arXiv:1103.2766. S.Galli, F.Iocco, G.Bertone, A.Melchiorri, Phys. Rev. D 84 (2011) 027302, arXiv:1106.1528. A. Natarajan, arXiv:1201.3939. G. Giesen, J. Lesgourgues, B. Audren and Y. Ali-Haimoud, JCAP 1212 (2012) 008 [arXiv:1209.0247]. J. M. Cline and P. Scott, JCAP 1303 (2013) 044 [arXiv:1301.5908].

[13] T. Bringmann and C. Weniger, arXiv:1208.5481 [hep-ph].

[14] T. Bringmann, X. Huang, A. Ibarra, S. Vogl and C. Weniger, arXiv:1203.1312 [hep-ph].

[15] C. Weniger, JCAP 1208 (2012) 007 [arXiv:1204.2797 [hep-ph]].

[16] E. Tempel, A. Hektor and M. Raidal, arXiv:1205.1045 [hep-ph].

[17] M. Su and D. P. Finkbeiner, arXiv:1206.1616 [astro-ph.HE].

[18] A. Hektor, M. Raidal and E. Tempel, arXiv:1207.4466 [astro-ph.HE].

[19] M. Su and D. P. Finkbeiner, arXiv:1207.7060 [astro-ph.HE].

[20] A. Rajaraman, T. M. P. Tait and D. Whiteson, arXiv:1205.4723 [hep-ph].

[21] A. Boyarsky, D. Malyshev and O. Ruchayskiy, arXiv:1205.4700 [astro-ph.HE].

[22] N. Mirabal, arXiv:1208.1693 [astro-ph.HE].

[23] A. Hektor, M. Raidal and E. Tempel, arXiv:1208.1996 [astro-ph.HE].

[24] C. Weniger, presentation at the FERMI meeting for alternative observations, 25 Jul 2013. 